\title{
HMGBI: a potential target of controlling pulmonary inflammation in people living with HIV
}

\section{Opinion}

The Acquired immunodeficiency syndrome (AIDS) is commonly known as the result of human immunodeficiency virus (HIV) infection. HIV attacks the human immune system, including CD4+ T lymphocytes, macrophages and dendritic cells, etc. The clinical manifestations after HIV infection are the continuous decrease of CD4+ T lymphocytes, which eventually leads to the deficiency of human immune function, leading to various opportunistic infections and the occurrence of tumors. ${ }^{1}$ According to the annual UNAIDS World AIDS Report in Geneva, Switzerland, an estimated 37.9 million people worldwide are living with HIV (about five out of every 1,000 people are infected with HIV), only 23.3 million are receiving antiretroviral therapy and 770,000 die from HIV-related diseases in 2018. Therefore, HIV/AIDS remains an important public health problem that poses a serious threat to human health.

AIDS combined with pulmonary infection is one of the most common diseases in opportunistic infections, accounting for $50 \%-69.6 \%$ of opportunistic infections. ${ }^{2}$ The pathogenic microorganismincludes bacteria, fungi, Pneumocystis carinii, Mycobacterium, cytomegalovirus (CMV), etc.CD4+T lymphocytes of HIV-infected patients are progressively reduced or even disappeared, leading to cellular immune deficiency and increasing the probability of lung infection.Pulmonary opportunistic infections were rarely seen in patients with CD4+T lymphocytes $>500 / \mathrm{mm}^{3}$. When CD4+T lymphocytes were $>350 / \mathrm{mm}^{3}$, the clinical symptoms were similar to those of ordinary pulmonary tuberculosis patients.However, when CD4+T lymphocytes <200/ $\mathrm{mm}^{3}$, they were susceptible to Pneumocystis carinii, CMV, fungi, bacteria and mycobacterium atypia.There are many different kinds of lung infection, such as pneumocystis carinii pneumonia (PCP), CMV pneumonia, toxoplasma infection,tuberculosis, atypical mycobacteria disease and candida pneumonia etc. Among these infections, PCP and tuberculosisoccur more frequently, they often exist as one or more pathogenic microorganism. These infections develop fast, bring to clinical diagnosis and treatment of great difficulties, to become one of the leading causes of death among AIDS patients. ${ }^{3,4}$

Previous studies ${ }^{3,4}$ showed that AIDS complicated with pulmonary infection was closely related to $\mathrm{T}$ lymphocyte subsets, phagocytic function of alveolar macrophages, levels of TNF- $\alpha$, IL-2 and the expression of TLR2 and TLR4 genes in lung tissue. These factors damage immune defense system of lung, leading to the recessions in the lung function of cilia clearance system, mucus gland secretion of the antimicrobial substances, serum complement components and pulmonary macrophage phagocytosis. Thus, pathogenic microorganisms are inhaled from the airway or ectopic focal infection pathogens are delivered through blood to alveolar, lung infection occurs.

Highmobilitygroupbox1 protein (HMGB1), named for its rapid migration in polyacrylamide gel electrophoresis, is a kind of DNAbinding non-histone protein with rich content in the nucleus. It is a new potential late-stage inflammatory mediator, which is involved in the pathogenesis of sepsis and an important late-stage inflammatory mediator of the lethal effect of endotoxin. It was reported to promote cell
Volume 8 Issue 5 - 2020

\author{
Yuexu Ding, Li Wang \\ Chongqing Public Health Medical Center, Chongqing, China
}

Correspondence: Li Wang, Chongqing Public Health Medical Center, 109 Baoyu Road, Shapingba District, Chongqing, China, Email 44366869@qq.com

Received: October 16, 2020 | Published: October 28, 2020

activation and release of proinflammatory factors..$^{5-9}$ HMGB-1 activates multiple signaling pathways through RAGE (receptor for advanced glycation end products) and/or TLR-induced intracellular signaling pathways, such as MAPkinase and JNK pathways, and subsequent nuclear translocalization of $\mathrm{NF}-\mathrm{\kappa B}$, leading to the expression of various leukocyte adhesion molecules, pro-inflammatory cytokines, and chemokines. ${ }^{10,11}$ It plays a key role in the process of sepsis, chronic obstructive pulmonary disease (COPD), lung infection, arthritis and gastrointestinal tumor. Moreover, it was also been found that signaling pathway of HMGB1/TLR4/NF- $\mathrm{KB}$ predominated in the pathogenesis of AIDS. ${ }^{12,13}$

Plasma HMGB1 level in people living with HIV was higher than that of HIV-negative control group, the mRNA expression level of HMGB1 and plasma HMGB1 level in AIDS patients with clinical symptoms were significantly higher than that of HIV-infected patients and HIVnegative control group. The mRNA expression level of HMGB1 and plasma HMGB1 levelin AIDS patients with poor efficacy after HAART treatment were also significantly higher than those in the group with good efficacy. When CD4+T cell count was lower than 200/L, HMGB1 content and HMGB1 mRNA expression level in peripheral blood mononuclear cells (PBMCs) were negatively correlated with $\mathrm{CD} 4+\mathrm{T}$ cell count, suggesting that HMGB1 may play an important role in the onset and progression of HIV/AIDS. ${ }^{13}$

Base on the growing evidences that showed pulmonary inflammation in AIDS patients and important effects of HMGB1 in lung infection, we propose that HMGB1 might be a key target of controlling pulmonary inflammation in people living with HIV.

\section{Acknowledgments}

None.

\section{Conflicts of interest}

The author declares that there are no conflicts of interest.

\section{Funding}

This work was supported by Chongqing Health and Family Planning Commission Traditional Chinese Medicine Science and Technology project (No. ZY201702049). 


\section{References}

1. Sangarlangkarn A, Merlin JS, Tucker RO, et al. Advance Care Planning and HIV Infection in the Era of Antiretroviral Therapy: A Review. Top Antivir Med. 2016;23(5):174-80.

2. Marcus JL, Leyden WA, Chao CR, et al. Immunodeficiency, AIDS-related pneumonia, and risk of lung cancer among HIV-infected individuals. Aids. 2017;31(7):989-93.

3. O'Connor J, Vjecha MJ, Phillips AN, et al. Effect of immediate initiation of antiretroviral therapy on risk of severe bacterial infections in HIVpositive people with CD4 cell counts of more than 500 cells per $\mu \mathrm{L}$ : secondary outcome results from a randomised controlled trial. Lancet HIV. 2017;4:e105-e12.

4. Sun J, Su J, Xie Y, et al. Plasma IL-6/IL-10 Ratio and IL-8, LDH, and HBDH Level Predict the Severity and the Risk of Death in AIDS Patients with Pneumocystis Pneumonia. J Immunol Res. 2016;2016:1583951.

5. Shu X, Zhang J, Wang Q, et al. Glutamine decreases intestinal mucosal injury in a rat model of intestinal ischemia-reperfusion by downregulating HMGB1 and inflammatory cytokine expression. Exp Ther Med. 2016;12(3):1367-1372.

6. Nehil M, Paquette J, Tokuyasu T, et al. High mobility group box 1 promotes tumor cell migration through epigenetic silencing of semaphorin 3A. Oncogene. 2014;33:5151-5162.

7. Zhu X, Messer JS, Wang Y, et al. Cytosolic HMGB1 controls the cellular autophagy/apoptosis checkpoint during inflammation. J Clin Invest. 2015;125(3):1098-1110.
8. Ojo OO, Ryu MH, Jha A, et al. High-mobility group box 1 promotes extracellular matrix synthesis and wound repair in human bronchial epithelial cells. Am J Physiol Lung Cell Mol Physiol. 2015;309(11):L1354-66.

9. Kim SY, Koh WJ, Park HY, et al. Down-Regulation of Serum HighMobility Group Box 1 Protein in Patients with Pulmonary Tuberculosis and Nontuberculous Mycobacterial Lung Disease. Tuberc Respir Dis Seoul. 2017;80(2):153-158.

10. Nogueira-Machado JA, de Oliveira Volpe CM. HMGB-1 as a target for inflammation controlling. Recent Pat Endocr Metab Immune Drug Discov. 2012;6(3):201-219.

11. Park JS, Svetkauskaite D, He Q, et al. Involvement of toll-like receptors 2 and 4 in cellular activation by high mobility group box 1 protein. $J$ Biol Chem. 2004;279(9):7370-7377.

12. Gougeon ML, Melki MT, Saïdi H. HMGB1, an alarmin promoting HIV dissemination and latency in dendritic cells. Cell Death Differ. 2012;19:96-106.

13. Tasca KI, Caleffi JT, Correa CR, et al. The Initial Months of Antiretroviral Therapy and Its Influence on AGEs, HMGB1, and sRAGE Levels in Asymptomatic HIV-Infected Individuals. Mediators Inflamm. 2016;2016:2909576. 